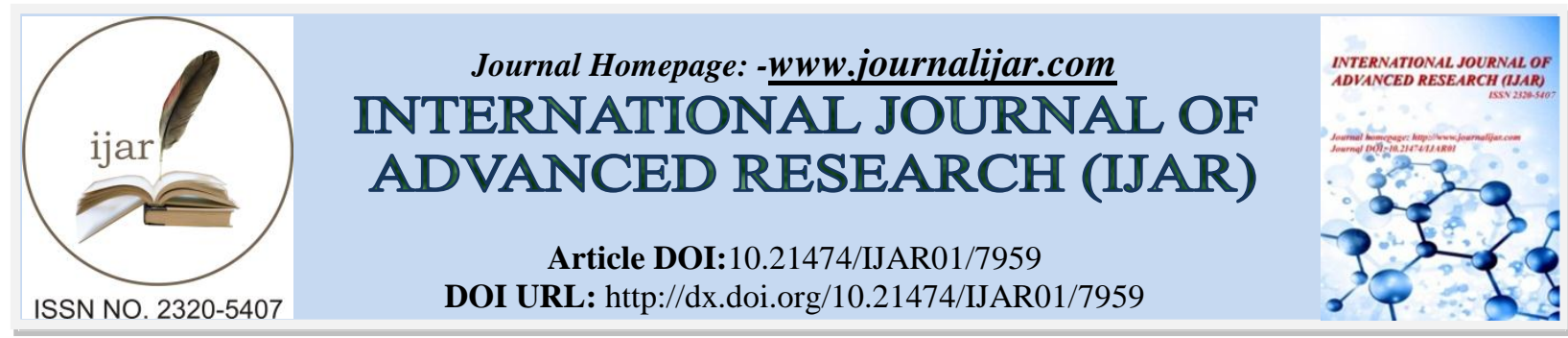

RESEARCH ARTICLE

\title{
INTELLIGENT IRRIGATION SYSTEM USING ARTIFICIAL INTELLIGENCE AND MACHINE LEARNING: A COMPREHENSIVE REVIEW.
}

1. Gandhinagar Institute of Technology, Gandhinagar, Gujarat, India.

2. Lalbhai Dalpatbhai College of Engineering, Ahmedabad, Gujarat, India.

3. Vishwakarma Government Engineering College, Chandkheda, Ahmedabad, Gujarat, India.

\section{Manuscript Info}

\section{Manuscript History}

Received: 17 August 2018

Final Accepted: 19 September 2018

Published: October 2018

\begin{abstract}
Agriculture automation is the main concern and emerging subject for every country. Where many types of research have been carried out like the implementation of fuzzy logic and Neuro- Fuzzy logic,automation using Expert systems and Artificial intelligence which led to great benefits.First and foremost, this paper broaches the subject of Artificial intelligence, machine learning, and embedded system. It further discusses the blending of AI and embedded technology in the agriculture sector. There are still some areas of problems which are causing the problems to agriculture field like Crop diseases infestations, lack of storage management, pesticide control, weed management, lack of irrigation and water management. In the Country like India where the water is one of the major problems for the people in agriculture sectors, and the government is trying to provide more and more support to implement automation in irrigation and agriculture. As the review suggests, The automation can be achieved along with smart embedded system by using the Arduino and Raspberry pi3 with the temperature and moisture sensor by deploying the machine learning algorithms and developing essential IoT(Internet of Things). As the world is more turning toward the online storage resources cloud computing is the major choice for data storage and management derived from the sensors and easily accessible from the user'sdevices. The automaton in agriculture with the implementation of the embedded system is also a pivotal topic for the crop prediction, evapotranspiration process. Evapotranspiration process is imperative for maintaining the stability in the hydrologic cycle, sustainable irrigation method, and water management. The paper discusses penetration of AI and embedded systems in agriculture sector via discussing past breakthroughs. The problem of water usage among the farmers leads to the smart irrigation system which will also result in the efficient use of water resources. The irrigation system proposed is fully automated and easily accessible method which will be beneficial to the agriculture automation to future scope.
\end{abstract}

Copy Right, IJAR, 2018,. All rights reserved. 


\section{Introduction:-}

Over the years, science and technology have taken a considerable amount of leap in the lives of humans. We as mankind have been resting on technology whether it is tangible or indirect. With the advent of technology in this digital world, we humans have pushed our limit of the thinking process and are trying to coalesce normal brain with an artificial one. This continuing exploration gave birth to a whole new field 'Artificial intelligence'. The term artificial intelligence was brought forth in front of the world by John McCarthy in 1956. It is the process by which a human can make an intelligent machine. AI comes under the domain area of computer science which can be able to discern its milieu and should thrive to maximize the rate of success. AI should be able to do work based on past learning. The decision process involved with AI should response quickly as accurate as it can. The scientific significance of AI is to program machines such that it can do work in which human intelligence is involved. AI can be phrased as "capability to hold two polar ideas in mind remain able to function and articulately take a complex decision."

AI has penetrated in medical science, education, finance, agriculture, industry, security, and many other sectors. Implementation of AI involves learning process of machines. This brings us to a sub-domain in this AI field "Machine learning". The sole purpose of machine learning is to feed the machine with data from past experiences and statistical data so that it can perform its assigned task to solve a particular problem. There are many applications which exist today which includes analyzing of data from past data and experience, speech and face recognition, weather prediction, medical diagnostics. As scientists advanced in AI, they felt a dire need to find a system if applied to a machine, it can learn itself. Apart from rudimentary problem-solving techniques, there was no method before machine learning that can solve complex problems with the help of simpler algorithms. It is because of machine learning that the domain of big data and data science has evolved to such a great extent. Machine learning is a mathematical approach to build intelligent machines.

As AI stimulated, many new logics and method were invented and discovered which makes the process of problemsolving more simple. Such methods are listed below.

1. Fuzzy logic

2. Artificial neural networks (ANN)

3. Neuro-fuzzy logic

4. Expert systems

Among all of these, the most widely used and constantly applied method for research purposes is ANN. Our human brain is the most complex part of the body. Based on the inter linked neural networks, electric signals traverses through the neurons with the help of axons. Synapses which are at the end of each node passes the signal ahead. ANN method was invented by keeping in mind the same concept of the working of the human brain. There are various algorithms of this approach. Based on the application, the correct algorithm is to be used. ANN is a taskbased method which tells the system to operate based on some inbuilt task rather than a conventional computational programmed task. The architecture of ANN consists of three layers :

1. Input layer

2. Hidden (middle) layer

3. Output layer (Figure - 1)

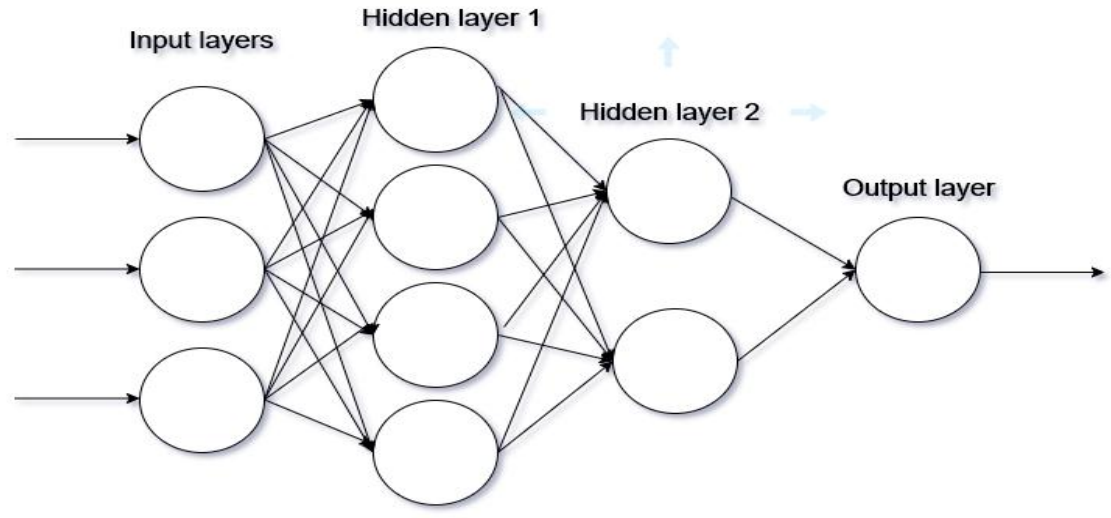

Figure 1:-Artificial neural network layers 
Moreover, Artificial intelligence and machine learning are mostly hypothesis and theories. These are programming and algorithms. For the implementation of these algorithms and logic based concepts, there should be a hardwaresoftware interface. The system through which this can be achieved is 'Embedded systems'. Embedded systems are hardware built systems consisting of memory chips with custom software programmed in it. (Figure - 2)

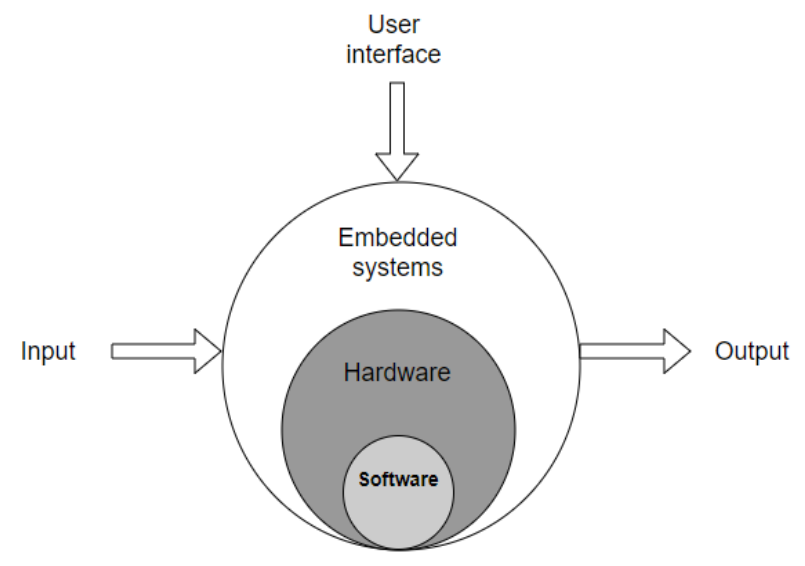

Figure 2:-Embedded systems

This paper encompasses the links which make embedded systems and AI coherent with the agriculture sector. Implementation of AI and expert systems in agriculture is a narrowly defined subject. This topic was discussed by Mckinion et al. back in 1985 (1985).Agriculture is the essential part of any country. At present South Korea, China, North America are investing trillions of money for development in the agriculture sector and implementing more advance technologies. The population is increasing at a very high rate which is directly related to the increase in the demand for food. India is a rich source for food crops and especially for species. The agriculture sector is one of the most sensitive sectors of the Indian economy, supporting all other sectors and spreading its importance in farreaching areas. With the advent of technology in other industries, it is a very crucial point to implement automation in agriculture. In India, farmers perform a traditional method for agriculture which satisfied the needs before,but as the world is moving with the fast speed and the population in India is increasing exponentially, it is very hard to reassure the demands. As population increases the demand for food increase and the call for agriculture automation rises. In recentyear's semi-automated methods have grown and served the rising demands but this is not sufficient enough to serve the whole purpose. A complete automated mechanism is what which will replace the traditional agriculture approach.

Automated mechanisms are implemented on the available traditional approach itself. Traditional agriculture methods like drip irrigation, sprinkler irrigation, direct pump irrigation,and many others need continuous field monitoring by the farmer. These systems can be automated using the Internet of Things controlled by a control system. The fully automated system understands the surrounding conditions and operates accordingly. The fresh water available on the earth is very less and effective use of it is the prime goal. The traditional methods used for irrigation was not very efficient and so to automate the irrigation system in order to monitor the field some additional technologies like intelligence is in much need so that the system can apply artificial intelligence to deduce the agriculture data and results and to predict future results and work correspondingly. Machine learning which is the part of artificial intelligence supports the Machine to Machine Communication (M2M). The whole mechanism learns the operation and operates as an artificial human brain. However, there are numerous problems such as crop loss, illiteracy among farmers about the crops and efficient use of water. This paper suggests the comprehensive solution to such problems related to agriculture with the help of AI-based irrigation systems. The proposed system will make efficient use of theories of AI and Machine learning and will manifest with the help of an embedded system. The system will decide the productivity of the soil, crops and irrigation system. The system will be designed in such a way that it can approach the maximum population of India.

\section{Literature survey}

Over the past 50 years, there has been a sustainable development in artificial intelligence due to its robustness in the application and is pervasive in every field. One such field is agriculture. Agriculture constitutes almost $30.7 \%$ of the 
world's population which is directly or vicariously attached to it. Almost 1.5 billion hectors of land (which is 11 percent of the total land area) are used for agriculture. Such a business faces many challenges on a daily basis and is not smoothly running. Some of the pith problems faced by farmers from seed sowing to harvesting of crops are as follows:

1. Crop diseases infestations

2. Lack of storage management.

3. Pesticide control

4. Weed management

5. Lack of irrigation and drainage facilities.

Computers and technology started penetrating in this sector from 1983 onwards. Since then, there have been many suggestions and proposed systems for betterment in agriculture from the database to decision making process. Filtering out every process, only AI based systems have proved to be the most feasible and reliable one. The AIbased method does notgeneralize the problem and gives a particular solution to a particular defined complex problem.

\section{Application of Artificial neural networks in crop prediction and evapotranspiration process.}

A method is suggesting the use of ANN algorithms for crop prediction in smartphones had been successfully tested in 2016 by researchers Ravichandran and Koteeshwari. A prediction model was developed. As mentioned above, the prediction model of this system had three layers. The efficiency of the model was dependent on the number of the hidden layers. First and foremost, the ANN model was built and trained using various algorithms such as Silva and Almeida's algorithms, Delta-bar-delta,Rprop, and various other to find the most favourable configuration. Trial and error method was implemented to choose the number of hidden layers. There should be a précised way to scrutinize the selection of some hidden layers because the prediction system's accuracy is dependent on the number of hidden layers. It was observed in the research that more the number of hidden layers in the ANN model; the more accurate were the prediction.

Since the purpose of the system was to make it handy for the farmers, it is developed on APK platform. The source code was written in Eclipse with Java codes in the backhand,and the algorithm was developed using Matlab and ANN toolbox. The whole file was then extracted on the Android platform so that it can be utilized by smartphones. Besides suggesting the crop to the farmer, the system also has the additional advantage of advising the farmer for the fertilizer to be used if the farmer wishes to use the crop of his choice. (Ravichandran and Koteshwari, 2016).(Figure $-3)$

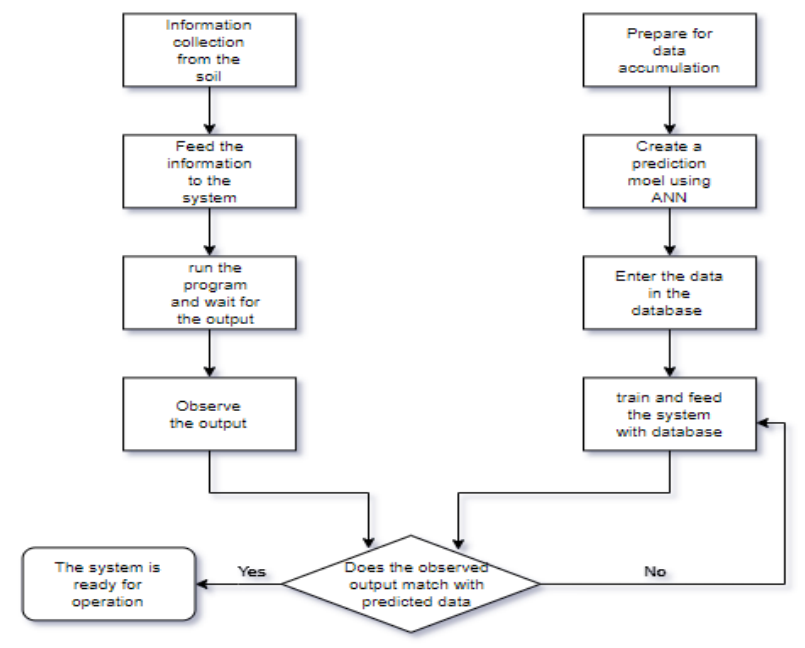

Figure 3:-Flowchart of ANN-based crop predictor using smartphones

Evapotranspiration process is imperative for maintaining the stability in the hydrologic cycle, sustainable irrigation method, and water management. There are more than 20 established method to determine ET which is dependent on several parameters. A profound study was carried out in the valley of Dehradun; India was assessing the importance 
of the addition of ANN in several techniques of estimation of ET. Researchers gathered monthly climate data from the Forest research institute (FRI) Dehradun for ET estimation. The methods on which the algorithms were applied were: 1. Penman-Monteith method 2. Levenberg-Marquardt backpropagation. It was observed that increasing the number of hidden layers in the system resulted in instability in the ET estimation. So, training function with optimum trial and error method is to be chosen for the overall optimized estimation of ET. It was observed that out of six training algorithms of ANN model, function 'trainlm' with 75\% data feed in it was precise and had the best number of neurons. Furthermore, there was a gauging between PM method and ANN model with the single layer feed forward back propagation algorithm. ANN model was designed and developed using Matlab. Six algorithms were conjured and assessed. As evapotranspiration is of vital importance in irrigation and water management, this research manifested the predictive prowess of ANN structure if implemented correctly. ( Nema et al., 2017).

Furthermore, also a method was created to discriminate weed from crops with the help of image analysis and neural networks. It had the accuracy of more than $75 \%$ without any beforehand plant information fed into the system. (Aitkenhead et al., 2003). Shahzadi et al. (2016) developed expert systems based smart agriculture system. The concept of IoT in this system was to send the data to the server so that actuators of the field should be able to take appropriate decisions. For that, the server should be intelligent enough to take decisions independently. This system consists of temperature, humidity, leaf wetness, and soil sensors. It only gives the information about the field and doesn't act on the irrigation process.

\section{Machine learning and smart embedded systems in the agricultural sector.}

India constitutes $4 \%$ of the fresh water available on the earth out of which, the farming sector consumes $80 \%$ of water. This is a matter of grave concern. It is imperative that the water used in the irrigation system should not be wasted. The only way to stop squalid of water in irrigation is to introduce a smart system which takes the toll of usage of water and alleviate the problems as articulately as possible. Many researchers and organizations have brought forth the concept of Machine Learning and Smart IOT based system for improving irrigation and water management in agriculture.

Machine learning had been employed for crop disease prediction in 2016. Taking into consideration the socio and economic vitality of agriculture in India, researchers Suyash and Sandeep developed a system which predicted grape disease beforehand. Any anomaly in the grape plant was noticedonly after it was infected and this had a considerable deteriorating effect on the whole vineyard. The system employed various sensors such as temperature sensor, leaf wetness sensors, and humidity sensors in the vineyard. These sensors send the data sensed to the database in the ZigBee server which is linked to the sensors. The server will store the data. The server is commissioned with a hidden Markov model algorithm in it. This algorithm is present to train the normal data sensed by the sensors and report any aberration in temperature, humidity or leaf wetness which can result in grape disease to farmer via SMS. Machine learning is blended in the system beforehand for astute deduction of disease in grapes. The additional advantage of this system is it also suggests the farmer pesticides and pacifies manual effort in the detection of disease. ( Patil and Thorat, 2016). While a similar method of machine learning was employed in monitoring the growth of Paddy crops. This system was developed for increasing the yield and productivity of paddy crops. It also proved to be cost effective and durable. ( Kait et al., 2007).(Figure -4)

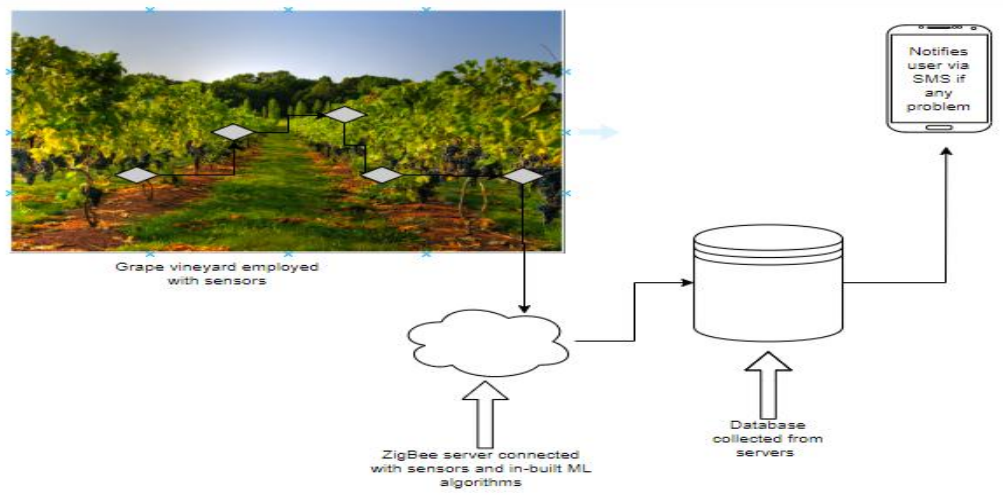

Figure 4:-Grape disease detection system using ML algorithms

In one of the research conducted in Ankara, Turkey, implementing IIS (intelligent irrigation system), numerous positive perks were observed such as less moisture and temperature stress on soil, efficient water consumption, and 
neglectinghuman intervention in case of flood irrigation. The developed system works on three units. Base unit (BU), Valve unit (VU), and Sensor unit (SU). The whole system is powered by solar panels. After the successful installation of every unit, BU will send the address to which the data is to be sent to SU. Sensors from the SU will sense the moisture content and send the detected data to a specific address in the BU. If required, BU will send a signal to VU so that it can calibrate the position of the valve in order to provide the soil with water. However, sitespecific use of automatic irrigation system took birth in the early $21^{\text {st }}$ century; this method proved to be a significant success as it reduced the cost, feasibility, and complexity of the developed system. Furthermore, the unit can be set up which transports the fertilizers and pesticides in the field using the same method. For that, new kind of sensors would have to calibrate for transmitting accurate information. ( Dursun and Ozden 2011).

Research has been conducted to test the ET based, ICT based, and IIS based technology. In Riyadh, a research was conducted in Wheat and Tomato field in which both sprinkler and drip irrigation method were employed and tested with ICT as well as IIS. A graph of water depth versus growth period of the crop (weekly) was plotted for all three methods. A concise observation concluded that IIS was far more feasible in a matter of water usage than ICT and ET based system. It skyrocketed the frugality of water usage from $18 \%$ to a whopping $27 \%$. (Al-Ghobari et al., 2011).

Also, Kodali and Sahu (2016) presented the use of Losant platform for monitoring the agriculture farmland and intimate the farmer via SMS or e-mail if any anomaly is observed by the system. Losing is a simple Iot based most powerful cloud platform. It offers real-time observation of data stored in it irrespective of the position of the field. Gutierrez et al. (2014) came up with an automated irrigation system which uses the GPRS module as a communication device. The system is programmed into a microprocessor-based gateway which controls the water quantity. It was proved that water savings were $90 \%$ more than the conventionalirrigation system. Kim et al. (2008) used a distributed wireless network for sensing and control of irrigation process from a remote location.

\section{Need for smart irrigation systems.}

Brief Report on water problems in India: India and China alone constitute 2.7 billion people living under the stress of water shortage. Out of overall water consumption, $70 \%$ is consumed in the agricultural process. Remaining is used in infrastructural pipelines and other miscellaneous works. Water leakage is inevitable and uncontrollable in cities. Water demand will shoot up by $50 \%$ shortlyand this fact cannot be vetoed away. The farming fraternity is the only option in which water usage can be optimized by employing smart irrigation systems.By inculcating smart irrigation system, wastage of water can culminate to a great extent can abridge water consumption by $20 \%$. ( Gupta et al., 2016).

\section{Smart irrigation systems}

There is a major problem of water wastage and a dearth of water in conventional irrigation methods employed. To give an example, Egypt faces a problem of water distribution from the Nile river with neighbouring countries. Ample research had been carried out to solve the problems faced in the irrigation, process. Many companies have developed a sensor-based smart irrigation system. These systems have been developed for optimal water usage, monitoring of water pollution, and to take care of some other grave problems. Soil moisture and temperature sensors interact directly with embedded components in the field and take care of required water distribution among crops without farmer's interaction.

Wall and King (2004) came up with a smart system which controlled valves of sprinklers with the help of temperature and moisture sensors deployed in the field. However, this system did not consider the water pollution problem. Miranda et al. (2003) came up with a distributed irrigation system which works on soil water measurement. M2M (machine-to-machine) technology which allows machines to interact with each other autonomously and store the data directly in a cloud-based server online. This M2M technology is in an incipient stage and is developing steadfastly. Shekhar et al. (2017) developed a technology which allows machines to communicate themselves. Yang et al. (2007) also developed a complete sensor-based intensive irrigation method which is self-organizing. This system constructed a bottom and upper layer. Pawar et al. (2018) tried to demonstrate a prototype of the small-scale smart irrigation system. M. Savitha and Uma Maheshwari (2018) considered only automation and IoT I in their quest for an intelligent irrigation system.

So far, there hasn't been such advent which allows complete freedom of human intervention. This paper tries to bring forward a method through which with the help of AI and embedded technology which eliminates the glitches emphasized in the past. 


\section{The proposed AI-based intelligent irrigation system.}

So far, there have been many advancements in precision agriculture. However, almost all the breakthroughs excludes the use of an intelligent machine to machine interaction in irrigation. Most of the systems fetched the data at a particular time and responded immediately controlling the valves in the field. There has not been any system which takes the decision based on past experiences and analyzingreal-time data. Till now, machine learning has only succeeded to abate crop yielding, crop disease detection, and crop management problems. There is no or meagre research in the field of machine learning technique which analyzes the soil moisture content based on past data fed and controls the irrigation process without any involvement of human work.

Temperature and moisture sensors will sense the soil condition at regular intervals and send the data to the microcontroller of the Arduino. Arduino has an inbuilt analog to digital converters which will convert input analog data to a digital one. An edge level hardware will be connected to Arduino. In this system, raspberry pi is connected. The converted signal will be sent to the raspberry pi. Machine learning based KNN algorithm is employed in the hardware. This algorithm is very simple and rudimentary. The sensed temperature and moisture values are then engulfed in the machine learning algorithm and based on the training values; Raspberry pi3 sends the signal to Arduino to start the pump for irrigation process. If the value corresponds to the value needed for the real-time soil requirement, then the pump will not get the command to start the process. Regardless of water supplied, every time the data is processed in the algorithm, it also updates the database. This database holds the information of sensor values and number of irrigation processes. This database uploads itself on a webpage cloud portal through which farmers can access the information. (Shekhar et al., 2017)

Comprehensive research on soil degradation due to natural as well as man-made factors should be carried out. A hidden Markov model should be designed to label and classify various factors affecting soil's health. There are government bodies like soil and water conservation division of the ministry of agriculture and the Indian Council of Agricultural Research (ICAR) which can help with the past statistical data. This accumulated data should be classified and labelled inside the neural network. By introducing ANN algorithms juxtaposing the KNN algorithms will keep the farmer updated about the condition of the soil.

(Figure-5)(Figure-6)

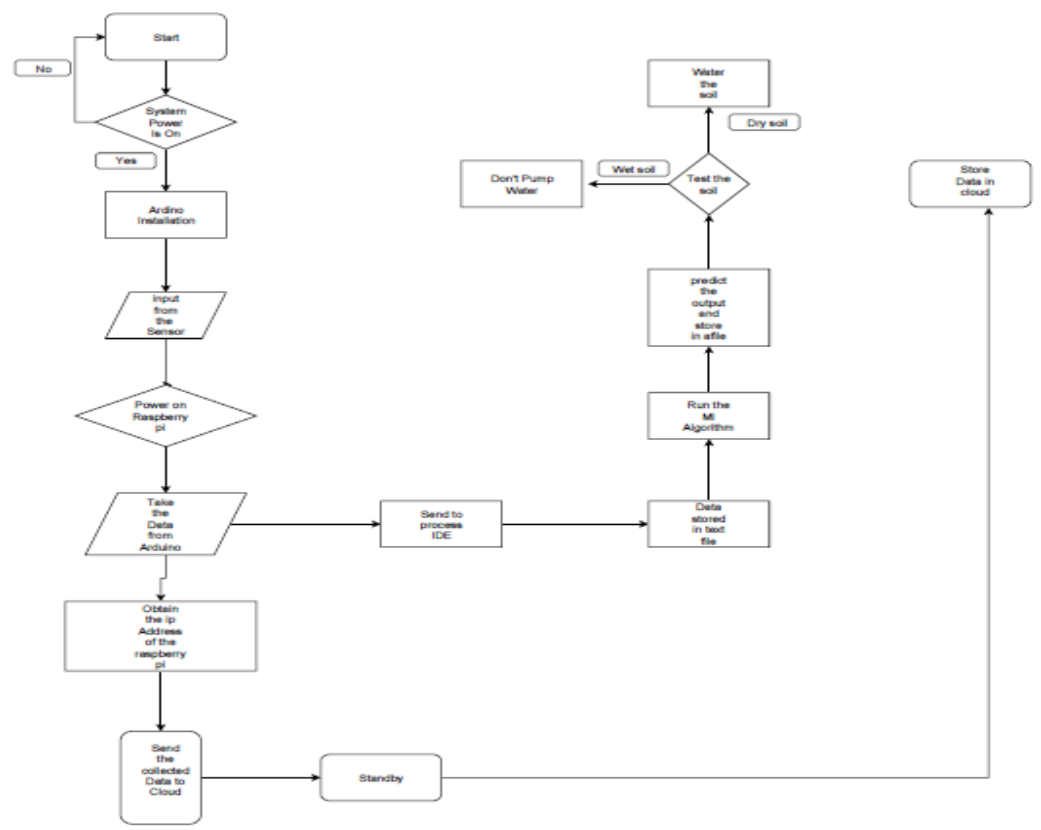

Figure 5:-A complete flowchart of IIS 


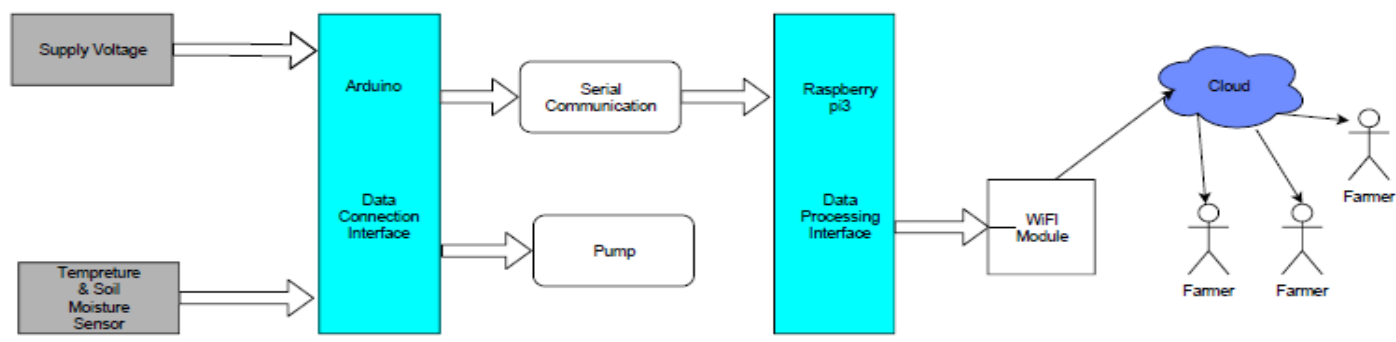

Figure 6:-Block diagram of proposed system.

\section{Conclusion and Future Work}

Agriculture monitoring is the much necessitate reducing human interventions in practice. Day by day demand for food is reaching its high peak and the without execution of the modern methods in agriculture it is very hard to achieve the increasing demand. Agriculture monitoring is the prime concern as it helps to reduce labour and increase the production. Artificial Intelligence has been implemented in crop selection and to help the farmer in the selection of the fertilizers. With the help of the database which the user has gathered and specified to the system, the machine communicates among themselves to decide which crop is suitable for harvesting and also the fertilizers which promote the maximum growth.

$\mathrm{AI}$ is also handed-down for water management and irrigation by using the artificial neural network (ANN) which helps to build a sustainable system. Water management is very important,and ANN is used to set the ET system. It is a veryeffective way to build an irrigation system which controls the amount of field water. Machine learning is a very trustful subject,and its application can create wonders in the field of agriculture. Machine learning is normally used to calculate crop yield and to calculate crop growth. Machine learning can also be implemented to know the crop disease. The data of the field is continuously sent to the server and monitored by the user. Humidity, wetness of the leave, colour pigmentation and many other parameters decide the plant's health. If there is any change in the value,the user will be informed on the web server itself. Machine learning learns from the past data and implements accordingly.

IOT marked its significance to help in the real-time monitoring of the data. The user can monitor the data of the sensors on the local host. IOT is mainly used in an intelligentwatering system where the moisture level of the soil is monitored,and if the moisture level reduces below the threshold value, the system starts the pump to water the field. Effective use of the available fresh water is essential and with the advancement in the technology and application of automation water crisis can be solved.

Traditional methods in agriculture have minor effects in this modern world. Water scarcity and flooding both are the major problems farmers are facing using the traditional approach. Many loop holes in this system and the alarming need to protect the agricultural land leads to the development of agriculture automation. This paper represents an idea to make a system with the use of sensors, IOT and machine learning to automate the traditional practices in agriculture. The idea is to implement ANN and KNN method both in the same system. Raspberry Pi module has algorithms of the KNN methodwhich helps the machine to learn from the previous data set of crop selection, crop growth and crop yield to give the data to the farmer. Whilethe ANN method continually measures the soil health and sends the data to the cloud. Use of more layers gives better results and more segregated data. The data is stored on the web server which then can be visible to the farmer on the web browser and on the mobile phone too. Real-time monitoring of all the data given by the sensors and machines is possible with the use of IOT. The HTML code is dumped into the Wi-Fi module and as a result, farmer can view the data on the local host of the web browser. This paper discusses various ideas where advanced technologies can be used to reduce the efforts in agriculture. In future, if the proposed idea is carriedforward, then it would lead to a great reduction in labour, easy agriculture practices, effective use of water resources and less power consumption. 


\section{Declaration}

\section{Authors Contribution}

All the authors make a substantial contribution to this manuscript. KJ, AD and PP participated in drafting the manuscript. KJ and $\mathrm{AD}$ wrote the main manuscript; all the authors discussed the results and implication on the manuscript at all stages.

\section{Acknowledgements:-}

The authors are grateful to Gandhinagar Institute of Technology, Lalbhai Dalpatbhai College of Engineering and Vishwakarma Government Engineering College for the permission to publish this research.

\section{Availability of data and material}

All relevant data and material are presented in the main paper.

\section{Competing interests}

The authors declare that they have no competing interests.

\section{Funding}

Not Applicable

\section{Consent for publication}

Not applicable.

\section{Ethics approval and consent to participate}

Not applicable.

\section{References:-}

1. Aitkenhead, M.J., Dalgetty, I.A., Mullins, C.E., McDonald, A.J.S., Strachan, N.J.C. 2003. Weed and crop discrimination using image analysis and artificial intelligence methods. Computers and Electronics in Agriculture, 39(3), 157-171.

2. Al-Ghobari, H.M., Mohammad, F.S. 2011. Intelligent irrigation performance: evaluation and quantifying its ability for conserving water in arid region. Appl Water Sci. 1:73-83

3. Dursun, M., Ozden, S. 2011. A wireless application of drip irrigation automation supported by soil moisture sensors. Scientific Research and Essays Vol. 6(7), pp. 1573-1582.

4. Encinas, C., Ruiz, E., Cortez, J., Espinoza, A. 2017. Design and implementation of a distributed IoT system for the monitoring of water quality in aquaculture. 2017 Wireless Telecommunications Symposium (WTS). 1-7.

5. Ganjegunte, G.K., Sheng, Z., Clark, J.A., 2012. Evaluating the accuracy of soil water sensors for irrigation scheduling to conserve freshwater. Appl Water Sci. 2:119-125.

6. Gupta, A., Mishra, S., Bokde, N., Kulat,K. 2016. Need of Smart Water Systems In India. International Journal of Applied Engineering Research. 11(4), 2216-2223.

7. Gutiérrez, J., Medina, J.F.V., Garibay, A.N., Gándara, M.A.P. 2014. Automated Irrigation System Using a Wireless Sensor Network and GPRS Module. IEEE Transactions on Instrumentation and Measurement. 63(1), $1-11$.

8. Kait, L.K., Kai, C.Z., Khoshdelniat, R., Lim, S.M., Tat, E.H. 2007. Paddy Growth Monitoring with Wireless Sensor Networks. International Conference on Intelligent and Advanced Systems, IEEE, 966-970.

9. Kim, Y.J., Evans, R.G., Iversen, W.M. 2008. Remote Sensing and Control of an Irrigation System Using a Distributed Wireless Sensor Network. IEEE Transactions on Instrumentation and Measurement, 57(7), 13791387.

10. Kodali, R.K., Sahu, A. 2016. An IoT Based Soil Moisture Monitoring on Losant Platform. 2nd International Conference on Contemporary Computing and Informatics, IEEE. 764-768.

11. McKinion, J.M., Lemmon, H.E. 1985. Expert systems for agriculture. Computers and Electronics in Agriculture. 1(1), 31-40.

12. Nema, M.K., Khare, D., Chandniha, S.K., 2017. Application of artificial intelligence to estimate the reference evapotranspiration in sub-humid Doon valley. Appl Water Sci. 7:3903-3910.

13. Patil, S.S., Thorat, S.A. 2016. Early detection of grapes diseases using machine learning and IoT. Second International Conference on Cognitive Computing and Information Processing (CCIP), IEEE, 1-5. 
14. Pawar, S.B., Rajput, P., Shaikh, A. 2018. Smart Irrigation System Using IOT And Raspberry Pi. International Research Journal of Engineering and Technology. 5(8), 1163-1166.

15. Ravichandran, G., Koteshwari, R.S., 2016. Agricultural crop predictor and advisor using ANN for smartphones. IEEE, 1-6.

16. Savitha, M., UmaMaheshwari, O.P. 2018. Smart crop field irrigation in IOT architecture using sensors. International Journal of Advanced Research in Computer Science. 9(1), 302-306.

17. Shahzadi, R., Tausif, M., Ferzund, J., Suryani, M.A. 2016. Internet of Things based Expert System for Smart Agriculture. International Journal of Advanced Computer Science and Applications. 7(9), 341-350.

18. Shekhar, Y. Dagur, E. Mishra, S., Tom, R.J., Veeramanikandan, M., Sankaranarayanan, S. 2017. Intelligent IoT Based Automated Irrigation System. International Journal of Applied Engineering Research. 12(18), 73067320.

19. R. W. Wall and B. A. King, - Incorporating Plug and Play Technology into Measurement and Control Systems for Irrigation. Management,\| 2004, Ottawa, Canada August 1 - 4, 2004, 2004.

20. Yang, H. Liusheng, W. Junmin, X. Hongli, -Wireless Sensor Networks for Intensive Irrigated Agriculture, Consumer Communications and Networking Conference, 2007. CCNC 2007. 4th IEEE, pp.197-201, Las Vegas, Nevada, Jan. 2007

21. Miranda F.R., Yoder R., and Wilkerson J.B., "A site-specific irrigation control system", presented at the ASAE Annu. Int. Meeting, Las Vegas, NV, Jul. 27-30, 2003. 